\title{
Ecosistema de emprendimiento e innovación en Cauca, Colombia. Experiencia desde el Centro de Desarrollo Tecnológico CreaTIC
}

\author{
Rivera Martínez, Wilfred Fabián ${ }^{1}$ \\ Canay Pazos, José Raúl ${ }^{2}$
}

\section{Resumen}

Este trabajo analiza la evolución de los emprendimientos de base tecnológica que conforman el Cluster CreaTIC, una apuesta caucana por consolidar un ecosistema de emprendimiento alrededor de la industria $\mathrm{TI}$ en el suroccidente colombiano como un sector clave para el desarrollo económico y social de la región. Aplicando herramientas metodológicas mixtas se realizaron entrevistas a Directores Ejecutivos en la historiadel Cluster, así como la revisión de 30 emprendimientos usando la técnica de análisis de conglomerados. En el ámbito organizacional se observa un proceso de emprendimiento alrededor de la industria TI caracterizado históricamente por una alta rotación de emprendimientos y un proceso de consolidación de empresas que coincide con una reforma a la ley que re-distribuye los recursos de las regalías de la renta minero energética en departamentos que tradicionalmente no recibían estos recursos. Esta investigación permite la agrupación en 4 categorías de empresas según la proximidad en las variables de medición, lo que posibilita una mejor intervención; asimismo se sistematizó la historia de la organización brindando pautas de acción y sugiriendo estrategias de aprendizaje y des-aprendizaje para aquellos territorios que apuntan al posicionamiento de actividades productivas estratégicas a partir de la tecnología y la innovación.

Palabras clave: Organizaciones; Empresas de Base Tecnológica; Cluster; Emprendimiento.

Recibido: 20.03.19 Aceptado: 20.06.19

1 Doctorando en Desarrollo Regional e Integración Económica de la Universidad Santiago de Compostela. Asesor en Estrategia Corporativa del Centro de Desarrollo Tecnológico CreaTIC en Cauca, Colombia. ceo@clustercreatic.com. https://orcid.org/0000-0003-2888-7929

$2 \mathrm{PhD}$ en Economía, investigador del Grupo Análise e Modelización Económica - IDEGA. Universidad de Santiago de Compostela. https://orcid.org/0000-0003-4994-7417 


\title{
Ecosystem of entrepreneurship and innovation in Cauca, Colombia. Experience from the CreaTIC Technology Development Center
}

\begin{abstract}
This paper analyzes the evolution of technology-based companies which are involved inCluster CreaTIC, an iniciative to consolidate the entrepreneurial ecosystem around the IT industry in southwestern Colombia as a key sector for the economic and social development of the region. Methodologically, we used mixed methodological tools and interviews to executive directors in the history of the Cluster, as well as data review of 30 startups using Analysis of Conglomerate. At the organizational level, there is a process of entrepreneurship around the IT industry characterized byits high rotation of companies and a process of consolidation that coincide with a reform of the law that redistributes the resources of the royalties of mining resources in departments that traditionally did not receive these resources significantly. This research allows 4 categories of startups according to the proximity in the measurement variables which allows a better intervention, we also systematized the history of the organization providing action guidelines and suggesting learning and de-learning strategies for those territories that point to the positioning of strategic productive activities based on technology and innovation.
\end{abstract}

Keywords: Organizations, Technology Based Companies, Cluster, Entrepreneurship.

\section{Introducción}

Cauca Región de Emprendimiento Avanzado en TIC -CreaTIC-surge como respuesta a la necesidad de adaptar una ciudad, Popayán, y una región, el Cauca, Colombia, que había ingresado al siglo XXI con una estructura económica clásica y con un índice de disolución mayor al de constitución de sociedades, a la nueva realidad económica y social.

CreaTIC se originóen 2002 ,inicialmente bajo la marca Parquesoft Popayán, fruto del esfuerzo mancomunado de diferentes sectores (la academia, el estado y el sector productivo de la región) en un momento de caída del crecimiento industrial en el Cauca, que solo en diez años había pasado de 34,8 al 5,4 por ciento(Bucheli, 2005:75). No existía la infraestructura industrial que se requería para la población profesional que generaban sus instituciones educativas en las áreas del conocimiento de la informática y la gestión, lo que generaba una fuerte migración de sus profesionales, agravando el problema de migración interna existente(Universidad Externado de Colombia, 2019)

Quince años después, el Cluster es una opción de emprendimiento para los jóvenes de la región,se fortalece con más de 70 emprendimientos 
atendidos,50 empresas de base tecnológica que tienen diferentes áreas de actuación y que hacen necesario iniciar un proceso de análisis de los resultados que se han obtenido. La organización lidera la consolidación del ecosistema de emprendimiento en el sur-occidente colombiano a partir del estudio, análisis y explicación de las diferentes relaciones complejas entre instituciones y personas emprendedoras con sus entornos académicos, sociales, políticos y económicos (OECD, 2014)

Para analizar las dinámicas que han dado origen y explican la naturaleza del ecosistema de emprendimiento e innovación en el Cauca, se ha planteado un trabajo de investigación mixto. Por un lado, se presenta un apartado cuantitativo, a partir del análisis de conglomerados de los emprendimientos atendidos, que pretende identificar grupos afines entre los emprendimientos que permitirá mejorar los análisis que se realizan sobre sus necesidades, expectativas e impactos en el territorio. Además, se ha abordado un apartado cualitativo a través de una serie de entrevistas realizadas a los cuatro directores que CreaTIC ha tenido desde su inicio, lo que permite profundizar en la comprensión de los resultados obtenidos. Este enfoque mixto, como señala Hernández (2014), logra un mayor entendimiento del fenómeno bajo estudio, así como el documentar parcialmente la intrahistoria del Cluster.

Desde la perspectiva teórica es necesario abordar conceptos que se imbrican para explicar la evolución de las dinámicas de emprendimiento e innovación en el Cauca. En primera instancia se presenta una revisión de las redes informales, elemento importante en los procesos de agregación de valor en organizaciones basadas en conocimiento para, posteriormente, analizar las principales características de un ecosistema de emprendimiento e innovación descritas en el ámbito literario.

\section{Redes informales en el emprendimiento}

Puesto que las empresas no son sistemas aislados, sino sistemas que son, en gran medida dependientes de sus relaciones con el entorno, se puede señalar la existencia de un capital relacional que se nutre de dichas relaciones. Esta dimensión incluye el valor que se genera a partir de las conexiones con los actores de interés tanto a nivel interno como externo, cuando estas conexiones evolucionan hacia relacionamientos que agregan valor entonces hablamos de capital relacional. Este capital está compuesto por el conocimiento que subyace entre la organización y sus grupos de interés.

Para Sveiby (1997)está dimensión tiene una orientación más fuerte hacia el entorno dado que involucra elementos como las marcas, el top of mind $y$ el top of heart, que desembocan en la reputación que una organización construye y presenta ante la sociedad. Algunos de estos elementos pueden ser protegidos legalmente y aunque en otros casos no procede un mecanismo legal de protección, resultan determinantes para los logros empresariales. Cabe destacar que las inversiones en este tipo de elementos implican un alto nivel de riesgo en virtud de la dificultad para calcular los retornos en términos financieros.

Para los efectos de esta investigación se concibe una startup como una institución humana diseñada para crear un nuevo producto o 
servicio bajo condiciones de extrema incertidumbre(Ries, 2011). En principio son más evidentes los relacionamientos establecidos con agentes vinculados ala startup en términos de proveeduría y demanda, y aunque pareciera que estas son las únicas relaciones, las redes personales del emprendedor resultan ser fundamentales para su empresa especialmente en las primeras etapas(Ostgaard \& Birley, 1994), de manera específica, el soporte de familiares y amigos resulta fundamental(Lechner \& Dowling, 2003).

En este sentido, vale la pena destacar que de manera empírica se ha evidenciado con los emprendedores que la familia provee soporte tanto emocional como financiero a las startups, en buena parte de los casos, familiares y amigos constituyen una fuente tanto de recursos económicos como de mano de obra que representa un ahorro significativo para el inicio de la startup. Si bien el trabajo realizado por familiares durante los primeros años del emprendimiento ayuda a compensar las restricciones financieras y a reducir los gastos, los emprendedores también destacan la lealtad y seguridad que ofrece el trabajo de un familiar dado que redunda en un menor esfuerzo de monitoreo y seguimiento de la actividad empresarial. Adicionalmente el soporte de estos actores que son importantes para el emprendedor también provee estabilidad emocional que beneficia la actividad de la startup (Brüderl \& Preisendörfer, 1998).

Existe una serie de factores que acompañan transversalmente la construcción del capital relacional y son percibidos por los emprendedores como catalizadores en sus emprendimientos: la reputación, conectividad, acceso a clientes, y conexión con proveedores, se plantean como elementos que generalmente aportan en la senda emprendedora de las empresas.

\section{Ecosistemas de emprendimiento e innovación}

Un ecosistema de innovación hace referencia a la red interconectada de empresas e instituciones que coevolucionan sus capacidades alrededor de un conjunto de tecnologías, conocimiento o habilidades y que trabaja cooperativa y competitivamente para el desarrollo de nuevos productos (Moore \& Garnsey, 1993). Las tres características distintivas de un ecosistema de innovación son: Las dependencias establecidas entre sus miembros, los objetivos y metas comunes, y el conjunto compartido de conocimiento $y$ habilidades. A escala global han sido ampliamente reconocidos y estudiados los ecosistemas de innovación en Silicon Valley, Cambridge e Israel (Adner, 2006).

Este estudio se centra en un tipo de ecosistema de innovación: el ecosistema de innovación basado en un hub el cual involucra a una organización que asume el liderato del ecosistema y ejerce una influencia considerable sobre las estrategias y destino de los miembros. Estos sistemas de innovación emergen gracias al importante contexto que brindan para el emprendimiento. Desde la perspectiva de lansiti \& Lavien (2004), la naturaleza del mercado objetivo y las grandes oportunidades en materia de apropiación tecnológica que caracterizan los ecosistemas de innovación estimulan la llegada de nuevos emprendimientos que a la postre incrementan el potencial para desarrollar innovaciones.

Para Alvarado y Rivera (2011) el emprendimiento simboliza la capacidad transformadora de las personas y se 
materializa en la creación de nuevos productos $\mathrm{o}$ en el mejoramiento significativo de los ya existentes para atender necesidades reales en la sociedad. Bajo esta óptica para que haya innovación en la empresa es necesario que el producto, el proceso, el método de comercialización o el método de organización sean nuevos o significativamente mejorados. Por lo anterior, el emprendimiento constituye un pilar clave de los ecosistemas de innovación en virtud de la capacidad de las personas para generar transformaciones que se cristalizan en productos que solucionan problemas en la sociedad.

Los emprendedores que habitan este ecosistema deben enfrentar desafíos especiales, en primera instancia deben gestionar sus emprendimientos a la vez que monitorean las fuerzas competitivas que emanan del ecosistema o de otros ecosistemas. Por otra parte, deben trabajar alineados a la visión, objetivos y estructuras definidas por el líder del ecosistema (hub). En este sentido se asume un doble trabajo al enfrentar las fuerzas competitivas propias de todo emprendimiento mientras que se aporta a la construcción colectiva de las directrices previamente establecidas por el líder del ecosistema, en efecto, no siempre sucede que el éxito de la organización líder conlleve al éxito de cada emprendimiento. En palabras de lansiti \& Lavien (2004), puede existir conflicto entre el significado del éxito para el ecosistema y lo que este signifique para cada miembro individual en dicho ecosistema. Por lo anterior es necesario que el emprendedor mantenga un balance entre sus acciones independientes y sus aportes al ecosistema.

Con el propósito de avanzar hacia la estructura metodológica de la investigación, se avizora la necesidad de determinar las causas que inciden en el crecimiento empresarial dado quela evolución del emprendimiento es una de las principales expectativas de quienes se atreven a cristalizar una idea de negocio. Existen posiciones y argumentos diversos entre autores que han tratado esta cuestión, Gibrat (1931)uno de los pioneros en el estudio del crecimiento empresarial, destacaba que el crecimiento de las empresas es un proceso estocástico originado por la acción de innumerables factores aleatorios que actúan de forma proporcional sobre el tamaño de las empresas, mientras que Albach(1967) argumenta que el crecimiento de la empresa es el resultado no de factores aleatorios, sino de una intención y determinación por parte de los empresarios y directivos que determinan su comportamiento. Ante posturas tan dispares, es posible concluir que el crecimiento empresarial está condicionado por factores diversos que podrían categorizarse como internos $y$ externos y que bien pueden constituir elementos que aceleran o frenan el crecimiento de las empresas.

\section{Algunas consideraciones metodológicas del estudio}

Analizar los aprendizajes de un proceso de construcción de un ecosistema de innovación en donde la piedra angular es el emprendedor, demanda el uso de técnicas cuantitativas para caracterizar los emprendimientos involucrados además de la incorporación de técnicas cualitativas que permitan la comprensión del relato de los agentes que han incidido en la historia de las dinámicas empresariales. 
Para Hernández (2014) el enfoque mixto de la investigación representa un conjunto de procesos sistemáticos, empíricos y críticos de investigación e implican la recolección y el análisis de datos cuantitativos y cualitativos, así como su integración y discusión conjunta, para realizar inferencias producto de toda la información recabada y lograr un mayor entendimiento del fenómeno bajo estudio.

La investigación toma como población de estudioa los actores de interés del Cluster, por un lado, los empresarios y por otro los Directores Ejecutivos que han estado al frente del mismo. Con los primeros se ha obtenido información cuantitativa para conocer con mayor detalle el tipo de empresas que habitan el ecosistema mientras que, con los directores, se realizaron entrevistas no estructuradas para conocer de primera mano las historias que permiten extraer aprendizajes y des-aprendizajes de la conformación del Cluster.

Según Blázquez (2006) a través de la literatura se ha podido constatar que la mayoría de los trabajos que analizan el crecimiento empresarial suelen considerar dicho fenómeno como factor determinante del incremento de la competitividad de la empresa, basándose dichos estudios en las consecuencias del crecimiento, más que en analizar las causas de dicho crecimiento. Ante este cuestionamiento, plantea factores internos como la antigüedad, el tamaño, la estructura de propiedad, la gestión del conocimiento; y externos como las ventas, los competidores, los proveedores, las mejoras tecnológicas, el acceso a financiación y el apoyo gubernamental. Todos los anteriores con una clara incidencia en el crecimiento de las empresas y con la capacidad de explicar las dinámicas que coadyuvan en la evolución de las mismas.

Bajo este entendido, para el análisis cuantitativo se tomó como referencia la base de datos de emprendimientos existente en el Cluster CreaTIC, de la cual se seleccionaron las treinta (30) empresas con mayor nivel de madurez

En cuanto a las variables se emplearon las cinco (5) recogidas en el cuadro 1 y que fueron tipificadas según los criterios descritos, lo que permitió que las variables quedasen estandarizadas en calificaciones de 1 a 4 . Estas variables han sido seleccionadas de la batería propuesta por Blázquez(2006) y comprenden aspectos que ya pueden ser medidos para el caso de los emprendimientos caucanos, estos son variables internas como la: i) Antigüedad, ii) número de empleados, iii) estructura de la propiedad, y variables externas, como son: i) ventas, ii) expansión. Estas variables que alimentan el análisis de conglomerados permiten una primera mirada al fenómeno del crecimiento en emprendimientos que intentan consolidar un ecosistema de innovación.

La batería completa de variables sin duda constituye un tema futuro de investigación para los efectos del análisis longitudinal de estos emprendimientos después de un periodo de tiempo que permita visualizar sus efectos a futuro (tabla1).El proceso estadístico se realizó empleando el software SPSS V22 for Windows. 


\section{Cuadro 1}

\section{Caracterización de las variables analizadas con empresarios de la industria TI caucana}

\begin{tabular}{|c|c|c|}
\hline Variable & Descripción & Criterios para tipificar variable \\
\hline Antigüedad & $\begin{array}{l}\text { Hace referencia al número de años } \\
\text { contados desde la fecha de su } \\
\text { registro en cámara de comercio del } \\
\text { Cauca }\end{array}$ & $\begin{array}{l}\text { Se dividió el grupo de } \\
\text { observaciones en bloques según } \\
\text { cuartiles. }\end{array}$ \\
\hline Empleados & $\begin{array}{l}\text { Hace referencia al número de } \\
\text { empleados que la empresa mantuvo } \\
\text { hasta el } 31 \text { de diciembre del año } 2015\end{array}$ & $\begin{array}{l}\text { observaciones en } 4 \text { bloques de } \\
\text { acuerdo a lo dispuesto en la ley } \\
905 \text { de Colombia (micro, } 1 \text { a } 10 ; \\
\text { pequeña } 11 \text { a } 50 \text {, mediana } 51 \text { a } \\
100 \text { y grande más de } 100 \text { ) }\end{array}$ \\
\hline Ventas & $\begin{array}{l}\text { Hace referencia al volumen total de } \\
\text { ventas en pesos colombianos que la } \\
\text { empresa tuvo desde el } 1 \text { de enero } \\
\text { de } 2015 \text { hasta el } 31 \text { de diciembre del } \\
2015\end{array}$ & $\begin{array}{l}\text { Se dividió el grupo de } \\
\text { observaciones en bloques según } \\
\text { cuartiles }\end{array}$ \\
\hline Expansión & $\begin{array}{l}\text { Hace referencia a los municipios } \\
\text { en donde la empresa vende sus } \\
\text { productos. }\end{array}$ & $\begin{array}{l}\text { Se dividió el grupo de } \\
\text { observaciones en bloques según } \\
\text { cuartiles. }\end{array}$ \\
\hline $\begin{array}{l}\text { Constitución } \\
\text { legal }\end{array}$ & $\begin{array}{l}\text { Hace referencia a su clasificación en } \\
\text { el registro de Cámara de Comercio. } \\
\text { Se establecen las opciones Sociedad } \\
\text { Anónima, Sociedad por Acciones } \\
\text { Simplificada, Sociedad Limitada }\end{array}$ & $\begin{array}{l}\text { Hace referencia a su clasificación } \\
\text { en el registro de Cámara de } \\
\text { Comercio. Se establecen las } \\
\text { opciones: Sin constitución, } \\
\text { Sociedad Anónima, Sociedad por } \\
\text { Acciones Simplificada, Sociedad } \\
\text { Limitada. }\end{array}$ \\
\hline \multirow{2}{*}{\multicolumn{3}{|c|}{ Fuente: Elaboración propia a partir de los postulados de Blázquez (2006) }} \\
\hline & & \\
\hline & Valor máximo & Media aritmética \\
\hline Antigüec & 19 años & 9.5 años \\
\hline Empleados & 19 & 11 \\
\hline Ventas & U\$250.000 & U\$ 100.000 \\
\hline Expansión & 12 municipios & 7 municipios \\
\hline
\end{tabular}

Fuente: Elaboración propia.

Como parte del análisis, se aplicó el análisis de conglomerados como técnica multivariante que agrupó elementos tratando de lograr la máxima homogeneidad en cada grupo y la mayor diferencia entre los grupos puesto que el propósito de esta técnica es la generación de grupos afines entre empresarios para mejorar los análisis que se realizan sobre sus particularidades, expectativas 
e impactos en la construcción de un ecosistema.

Como primer paso, se genera un dendrograma (figura 1) que indica que la opción que presenta mejores resultados en virtud de la claridad al representar bloques diferenciados es:
Método de conglomeración: Vecino más lejano, Intervalo: Distancia euclidea al cuadrado.

Posteriormente se calcularon los centroides, para identificar los vectores de medias de las variables usadas para cada cluster.

\section{Figura 1}

\section{Dendrograma de los empresarios de la industria TI caucana}

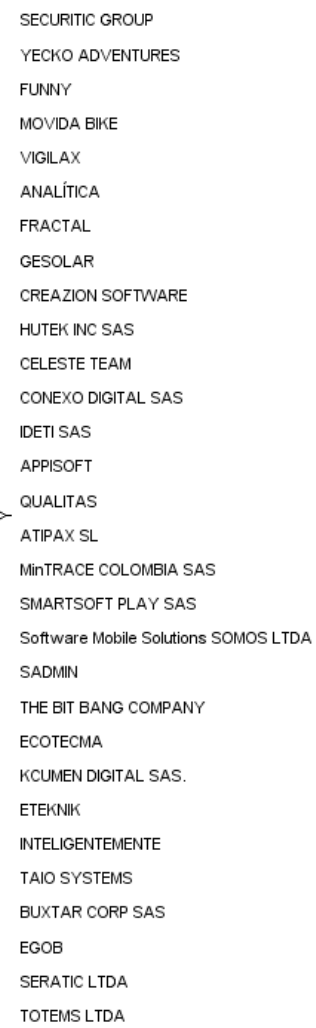

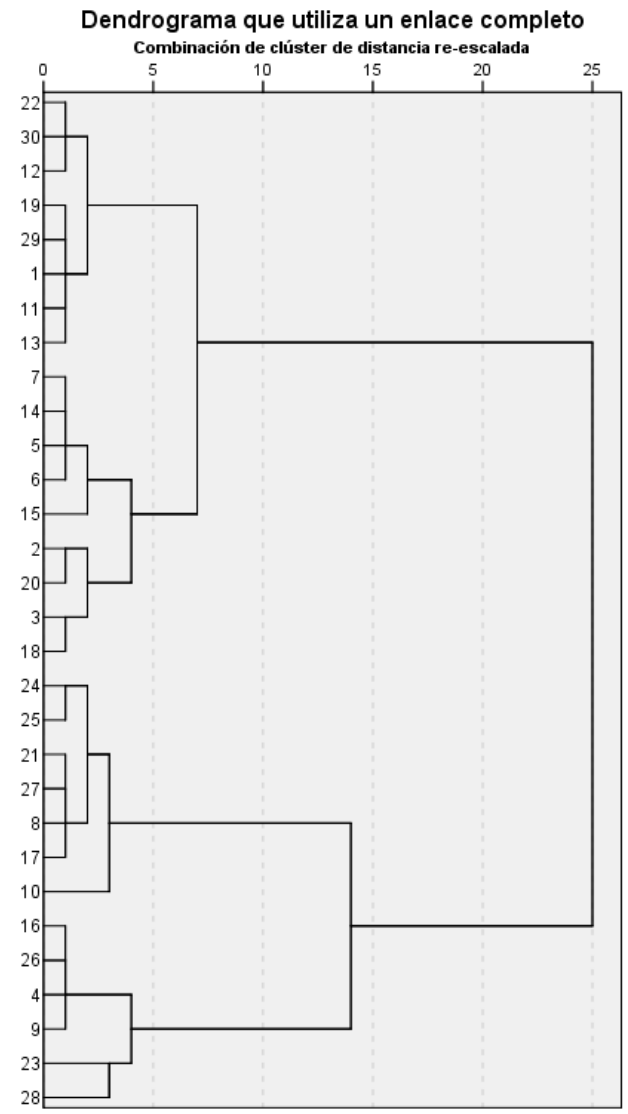

Fuente: Elaboración propia. 
El procesamiento realizado evidencia 4 categorías de empresas en el Cluster, los conglomerados dan cuenta de la existencia de empresas jóvenes, sostenibles y con la capacidad de mantenerse en el mercado. Por efectos nominales se asignan los rótulos de: Micro temprana, Micro avanzada, Pequeña temprana y Pequeña avanzada en virtud de los distintos grados de evolución que las caracterizan (tabla 2.

\section{Tabla 2}

\section{Caracterización de los empresarios a partir del análisis de Cluster}

\begin{tabular}{|c|c|c|c|c|}
\hline Conglomerado & Micro temprana & Micro avanzada & $\begin{array}{l}\text { Pequeña } \\
\text { temprana }\end{array}$ & $\begin{array}{l}\text { Pequeña } \\
\text { avanzada }\end{array}$ \\
\hline Empresas & $\begin{array}{l}\text { SecuriTIC, } \quad \text { Yecko, } \\
\text { Funny, } \quad \text { Movida, } \\
\text { Vigilax, Analítica, } \\
\text { Fractal, Gesolar. }\end{array}$ & $\begin{array}{l}\text { Creazion, Hutek, } \\
\text { Celeste, Conexo, } \\
\text { Ideti, Appisoft, } \\
\text { Qualitas, Atipax, } \\
\text { Mintrace }\end{array}$ & $\begin{array}{l}\text { InteligenteMente, } \\
\text { TaiO, Buxtar, } \\
\text { EGob, Seratic, } \\
\text { Totems. }\end{array}$ & $\begin{array}{l}\text { Smartsoftplay, } \\
\text { Sadmin, } \\
\text { Somos, } \\
\text { TBBC, } \\
\text { E c o t e c m a, } \\
\text { Kcumen, } \\
\text { Eteknik }\end{array}$ \\
\hline Ventas promedio & U\$ 12.000 & U\$ 20.000 & U\$ 70.000 & U\$ 130.000 \\
\hline Antigüedad (media) & 1,5 años. & 2 años. & 4 años. & 8 años. \\
\hline Empleados (Media) & 3 & 5 & 15 & 15 \\
\hline Extensión & 1 municipio & 3 municipios & 4 municipios & 10 municipios \\
\hline Constitución legal & Todas S.A.S & Todas S.A.S & Todas S.A.S & $\begin{array}{l}50 \% \text { S.A.S } \\
50 \% \text {. Sociedad } \\
\text { Ltda. }\end{array}$ \\
\hline
\end{tabular}

Fuente: Elaboración propia.

Si bien los niveles de madurez en términos de las variables analizadas reflejan 4 categorías de empresas en el Cluster, existen fuertes lazos de cooperación que permiten desarrollar ejercicios de comercialización asociativa entre las empresas. Las soluciones tecnológicas integrales son fomentadas desde el propio cluster y se cuenta con mecanismos de negociación claros y acuerdos de propiedad intelectual que dan confianza tanto a los empresarios como al mercado abordado.Como se ha mencionado, Sveiby (1997) establece el rol y la fuerza de las relaciones en la construcción de organizaciones fuertes, si bien los ejercicios asociativos son importantes en si mismo dada su capacidad de crear vínculos duraderos, cuando se conjugan estos ejercicios con el desarrollo de acciones comerciales conjuntas, no solo se crean alianzas, sino que se forjan acuerdos y reglas de juego claras que son fundamentales en el ejercicio de consolidar un ecosistema de innovación.

\section{Hacia la construcción de un ecosistema de emprendimiento e innovación alrededor de la industria TI: La historia del Cluster CreaTIC}

Las entrevistas realizadas a los cuatro (4) directores del Cluster CreaTIC permiten la realización de un recorrido histórico en la construcción de 
este ecosistema de emprendimiento e innovación en el Cauca.

El 18 de noviembre de 2002 se funda la corporación Parquesoft Popayán, no era un hecho menor, se creaba una organización que se dedicaría a la promoción y creación de empresas, en una ciudad donde el índice de disolución de sociedades era mayor que al de constitución de las mismas (Zambrano, entrevista 2018). Su idea, una necesidad desde hacía más de veinte años para un ciudad que había ingresado al siglo XXI con una industria clásica en servicios públicos, extracción de recursos naturales y manufacturas como única posibilidad de empleo y desarrollo para sus habitantes (Bucheli, 2003); era producto del esfuerzo mancomunado de diferentes sectores: del saliente gobierno municipal, de un grupo de profesores en la Universidad del Cauca, el Sena regional, del Crepic (Centro Regional de productividad e Innovación del Cauca), y de la incidencia de Parquesoft Cali.

Parquesoft Popayán se originaba en un momento de caída del crecimiento industrial en la ciudad, que solo en diez años había pasado de 34,8 al 5,4 por ciento; donde el crecimiento económico se ubicaba por debajo de la cifra nacional y en dondeel valor agregado en las industrias de alto conocimiento reportaba el más bajo en los últimos veinte años, situando al departamento en un nivel de competitividad bajo y en el lugar 20 del escalafón nacional que mide los 32 departamentos. Así el aporte de los campos emergentes como la informática y la tecnología, ni siquiera aparecía en las estadísticas (Zambrano, entrevista 2018).

Parquesoft se constituía en un contexto de necesidades y oportunidades que se debían de aprovechar para el desarrollo y el crecimiento económico de la ciudad. Así lo habían entendido sus creadores y después de más un año de dialogo interinstitucional entre los sectores educativos, gubernamentales y empresariales se fundaba con la esperanza de establecer una nueva industria en la economía del departamento. Su fundación históricamente significativa, para la economía local que exigía cambios drásticos en el pensamiento empresarial y en su constitución, reunía dieciséis organizaciones que participan activamente de la economía local, era la primera vez que se constituía una organización de tal carácter y que se reunía varios sectores por constituir una industria que la destacaba en el país. Las empresas e instituciones fundadoras pertenecían a los sectores educativos, empresarial y administración local, esto constituía una ventaja y oportunidad que en otras regiones las incubadoras no tenían.

La naturaleza y las condiciones en que se creó la Incubadora, fueron una apuesta de la academia, el sector productivo y el estado por generar un nuevo modelo de crear empresas que partiera de cambiar las condiciones mismas en que surgían. Participaban dieciséis organizaciones e instituciones, tanto públicas $(31 \%)$ como privadas (69\%).

\section{a.Los primeros años de auge}

En febrero del 2004 la Corporación se encontraba en un buen momento, había logrado incorporar el $96 \%$ de los procesos de incubación que se había propuesto, y había superado la cantidad de empleos y convenios que había proyectado en su primer año(Zambrano, entrevista 2018); tenía 
Ecosistema de emprendimiento e innovación en Cauca, Colombia. Experiencia desde el Centro de Desarrollo Tecnológico CreaTIC

Rivera Martínez, Wilfred Fabián y Canay Pazos, José Raúl

construido el plan estratégico y el de negocios de cada una las empresas incubadas. Sus locaciones contaban con instalaciones idóneas para alojar los empresarios, había pasado de ser una Corporación de un director a ser una comunidad con 140 personas. El banco de proyectos tenía cerca de cincuenta ideas de negocios para la industria del software, y se había posicionado con eventos propios y la participación a nivel local y nacional en conferencias divulgando el modelo de creación de empresas(Zambrano, entrevista 2018). En el ámbito económico habían vendido sus empresas cerca de mil millones de pesos (U\$ 330.000) superando lo presupuestado, y se estaban abriendo mercados internacionales; Se estaban ejecutando proyectos con el Servicio Nacional de Aprendizaje SENA y con el Departamento Administrativo para la ciencia y la tecnología COLCIENCIAS. Se encontraba en pleno despliegue su plan de trabajo que partía de ocho principios que se habían construido como valores que atravesarían sus prácticas siendo la Pasión, el Desprendimiento Económico, Trabajo duro, Innovación, Atrevimiento, Eficiencia, Sinergia $y$ Respuesta (Santacruz, entrevista 2018)

Al finalizar el año había pasado a tener 27 emprendimientos, ampliado sus instalacionesy se generaban a más de 155 empleos entre sus empresas, mejorando significativamente en convenios y locaciones para ofrecer mejores condiciones a las empresas.

\section{b. El estancamiento y transición}

Para el año 2006 se frenan las ventas y la ejecución de proyectos, y esto anunciaba una crisis. Aun así, se habían creado cuatro emprendimientos más y sus ventas eran de 1200 millones de pesos (U\$ 400.000), habían obtenido el Premio Innova con uno de sus emprendimientos con mayor potencial y tenían las primeras exportaciones con empresas incubadas (Santacruz, entrevista 2018).

En el 2007 empezaba su desaceleración económica vendiendo solamente un poco más de los 800 Millones de pesos (U\$ 260.000) incluyendo las empresas Ancla y había decaimiento institucional por la poca ejecución de proyectos que la soportaban. La Corporación comenzaba una crisis que la iba a minar en todos sus aspectos y que cobraría la primera Dirección Ejecutiva a pesar de que la había llevado a su primer auge. Se avizoraba que la misma fuerza de la política pública nacional que otorgó recursos para la creación de este tipo de organizaciones había generadodependencia de los recursos estatales para la operación, no se abrieron nuevas convocatorias de proyectos y empezaron a incumplirse los pagos al director y personal administrativo de la Corporación.

En menos de 2 años se presentó la renuncia de dos Directores Ejecutivos, los pasivos de La Corporación crecieron de manera considerable y sus fuentes de ingreso desaparecieron.

\section{c. La rebelión de los emprendedores}

Debido a su condición el emprendedor debe comenzar a alternar actividades para mantener su proyecto empresarial con las actividades de empleo para sostener sus gastos personales, así habían comenzado muchos de los que habían iniciado el proceso de la Corporación. Fueron estas prácticas las que les permitieron 
sobrevivir a los peores momentos de crisis en la Incubadora. Desde el comienzo, aunque contaban con unas mínimas condiciones para crear sus empresas, y esto les permitía comenzar a soñar, no podían renunciar a sus empleos. Sus propuestas desde temprano iban sustentadas y aunque fueron ignoradas, nunca desistieron de ellas. Así comenzaron manifestando sus ideas primero por medio del director y luego por representantes de la empresaancla que hacían parte de la Junta Directiva. Luego se darían cuenta que requerían sus propios representantes y órganos decisorios.

El 2011 finalizaría con el nombramiento del tercer director ad honoremque a nombre de los emprendedores y con los socios que habían quedado se dispondría en la refundación de la Incubadora. El tiempo de trabajo duro y de verse reducidos, de haberse ganado los espacios los había hecho conscientes que no bastaba con incidir en la política, sino que debían tomar el timón de la Incubadora. Con el nuevo esquema los emprendedores estarían no solo en equilibrio (Erazo, entrevista 2018) sino a la cabeza y vanguardia de la Corporación. Su rebeldía había sido consistente.

Con el nuevo liderazgo de los emprendedores se dieron al trabajo en dos frentes, en la prestación de servicios y en la formulación de proyectos para el desarrollo de las TIC en la región. Coincidía esto con el afianzamiento del Ministerio de las TIC y la creación del ambicioso plan nacional Vive Digital. Tanto el nuevo director como su equipo de colaboradores -emprendedores sobrevivientes- ad honorem empezaron la formulación de proyectos. Solo cuatro meses después lograban la aprobación de su primer proyecto con COLCIENCIAS, por un monto que superaba todo los gestionado anteriormente, más de mil doscientos millones de pesos (U\$ 400.000) (Samboní, entrevista 2018).

Enelámbitonacional se configuraba una política pública que abriría nuevas oportunidades, la redistribución de los ingresos del Sistema General de Regalías (SGR) que asignarían un 10\% para el Fondo de Ciencia, Tecnología e Innovación, permitiendo la financiación de proyectos regionales acordados entre las entidades territoriales y el Gobierno Nacional.

Tras esta decisión diferentes organizaciones que trabajan por el desarrollo científico y tecnológico del país empezaron a estructurar propuestas a fin de acceder a estos recursos. Tal es el caso de la Incubadora que estructuró una propuesta orientada al fortalecimiento de los procesos de creación de empresas de base tecnológica alrededor de la industria $\mathrm{TI}$ con actividades y recursos para consolidar un tejido empresarial de base tecnológica en un periodo de cinco (5) años.

En 2012 se aprobó el proyecto Cauca Vive Digital por 4.200 millones de pesos (U\$ 1.200.000) e iNNpulsa Colombia inyectó 340 millones de pesos (U\$113.000) a la iniciativa de encadenamientos productivos en la industria de TI caucana (Samboní, entrevista 2018). La Corporación con el control de sus emprendedores saldría de la crisis, gracias a la gestión ardua y decidida de un nuevo equipo de trabajo.

Los cambios logrados con el involucramiento de los emprendedores en los órganos directivos le dieron celeridad y efectividad a las asambleas y juntas directivas, formalizando los procesos y haciendo efectivos los órganos de decisión. La estructura se aligeraba para mejorar su efectividad y 
Ecosistema de emprendimiento e innovación en Cauca, Colombia. Experiencia desde el Centro de Desarrollo Tecnológico CreaTIC

Rivera Martínez, Wilfred Fabián y Canay Pazos, José Raúl

trabajar con lo que estaba contribuyendo a su refundación. La Corporación se hacía una incubadora de emprendedores para emprendedores (Samboní, entrevista 2018) y en este sentido las relaciones se hicieron horizontales entre los diferentes actores.

El 2013 inició con informe del director y presentó la evolución de su primer año de gestión, se renegociaron las deudas y pagaron gran parte de ellas. Se tenían instituciones aliadas que permitían avanzar. Se contaba conproyectos aprobados por más de dieciséismil millones de pesos (U\$ 5.300.000). Todo esto les permitía y exigía hacer una reforma estatuaria que se correspondiera y cerrara el ciclo de su refundación.

Así a finales de ese año se iniciaría el proceso de restructuración ampliando su objeto, pasando de ser incubadora de empresas de Software a de Base Tecnológica, lo que les permitía apostar por la diversificación de su producción; su quehacer ya no se reduciría a incubar empresas sino a potenciarlas con un proceso de aceleración, que les permitía, una vez creadas apoyarlas el proceso de crecimiento con la comercialización y el aumento de sus competencias. Las empresas ya no tendrían que sobrevivir su día a día, solas. A nivel de su concepción se determinó que la Corporación crearía su valor a partir de la producción de conocimiento y que sus modelos de incubación y aceleración estarían reglamentados. Nace entonces la Corporación de incubación y fomento de empresas de base tecnológica posicionando la marca CreaTIC acrónimo con el que se hace referencia a Cauca Región de Emprendimiento Avanzado en TIC. El proceso de ideación y creación de marca también estuvo a cargo de los propios emprendedores.
Se nombró una nueva Junta que lideraría la incubadora.A nivel estructural y organizacional se crearontres unidades estratégicas que soportan y viabilizan todos los procesos adoptando el modelo de unidades estratégicas de negocio.La UEN de creación de startups que asume los roles de incubación y aceleración de empresas de base tecnológica, la UEN del centro de desarrollo tecnológico, donde se acompañan las estrategias de innovación de las empresas, y la UEN de negocios estratégicos y marketing, en donde se gestan y desarrollan las acciones comerciales y trabajos conjuntos con aliados. Cada unidad con sus procesos, modelo de negocios y líderes que las agencian. Con esta nueva estructura y organicidad más el ambiente de cooperación interinstitucional y comercial, la construcción de un propio ecosistema para lograr sostenibilidad superando de esta manera el modelo y el proyecto inicial en su fundación, avanzó hacia una arquitectura de la innovación como propuesta propia.

En el 2015 iniciaron con una rendición de cuentas a la sociedad como una estrategia de trasparencia y de posicionamiento en la región como una verdadera oportunidad de desarrollo. Sus principales resultados: 50 empresas parte del Cluster,a nivel de la formación, trescientos emprendedores capacitados y en pleno proceso de aprendizaje empresarial para convertir sus ideas en negocios, más de 1.000 niños formados con su nuevo programa de súper desarrolladores y 500 docentes capacitados en el uso de las TIC e inclusión de la tecnología en la educación (Samboní, entrevista 2018). Se avanzó con 8 negocios propios que involucraban 31 municipios del departamento del Cauca, dando posibilidad del desarrollo de sus capacidades a más cien personas 
de ramas del conocimiento que van desde la psicología y educación hasta las ciencias fundamentales.

A nivel cualitativo se focalizaron los negocios en automatización del conocimiento, en tecnologías móviles, en internet de los objetos, y computación en la nube, con tres verticales fundamentales en su producción Agro y Agroindustria, Entretenimiento y Educación y Gobiernoy ya suman 30 aliados a nivel nacional e internacional.De esta manera en el 2016 la corporación comenzó un periodo de consolidación como industria convirtiéndose en un referente nacional en la creación de empleo y oportunidades, como en la gestión y ejecución de proyecto de alto impacto para el Departamento, convirtiéndose en una de las industrias que determinará el desarrollo de la Región, todo gracias a sus líderes emprendedores y su capacidad de reinventarse y trasformar la realidad para acometer los sueños.

Finalmente, todos estos esfuerzos y trabajos conjuntos con las empresas, se compilaron, documentaron y sistematizaron para participar de la convocatoria nacional de reconocimiento de actores del sistema de ciencia, tecnología e innovación; como resultado, CreaTIC obtuvo el reconocimiento como Centro de Desarrollo Tecnológico por los avances en términos de desarrollo de software, productos tecnológicos, aplicaciones y patentes estructuradas conjuntamente con las empresas. Con esto, se incrementa el acervo de capital intelectual de una organización que ha fundamentado su estrategia de crecimiento en su capital humano, estructural y relacional.

\section{Conclusiones}

El ecosistema de emprendimiento e innovación en el Cauca es un ejercicio territorial de aprendizaje que, aunque lejos de su etapa de madurez registra avances de interés. Así ha sido concebido tanto por quienes los orientan estratégicamente como por los emprendedores. Destacan el carácter territorial por que se trata de una apuesta por mejorar los niveles de competitividad de la ciudad y la región a través no solo de la creación de empresas de base tecnológica en la industria $\mathrm{TI}$ sino a través de la oferta a todo el aparato productivo de soluciones tecnológicas innovadoras y pertinentes al contexto local.

CreaTIC como organización tipo Hubque lidera el sector empresarial del ecosistema es una organización que aprende de su historia. En sus primeros años el apalancamiento con recursos del estado colombiano por la vía de proyectos generó una excesiva dependencia que acostumbró a los equipos de trabajo a formular y ejecutar proyectos. Cada vez que se presentan cambios de Gobierno y alteraciones a la política pública, este tipo de organizaciones no se adecuaba a las nuevas directrices nacionales. Hoy día se aprovechan las oportunidades que en materia de gestión de recursos trae la priorización de las TIC como eje transversal del desarrollo y la competitividad para el País. CreaTIC se apalanca en la gestión de recursos públicos por la vía de proyectos y aprovecha esta oportunidad para estructurarse con unidades estratégicas de negocio que tienen el equipo, los recursos y el know how suficiente para abordar nichos de marcado y desplegar una estrategia comercial que evidencia la cohesión y el trabajo conjunto. El Cluster es en si mismo una organización emprendedora que formula, ejecuta y se fortalece internamente con un portafolio 
Ecosistema de emprendimiento e innovación en Cauca, Colombia. Experiencia desde el Centro de Desarrollo Tecnológico CreaTIC

Rivera Martínez, Wilfred Fabián y Canay Pazos, José Raúl

de productos que ofrece al mercado.

El trabajo colaborativo ha sido un factor clave en el desarrollo de este ecosistema. Las tareas han sido planificadas y se han realizado ejercicios piloto de índole comercial que no solo dinamizan la estrategia de asociatividad, sino que permiten victorias tempranas que dan cuenta de los esfuerzos realizados en materia de innovación. Los actores involucrados realizanperiódicamente ruedas negocio, proyectos de innovación e investigación, misiones exploratorias, sesiones de trabajo con formuladores de política. Estos ejercicios permiten no solo estrechar lazos de cooperación, sino también ampliar el portafolio de servicios y visibilizar los logros comerciales de las empresas.

El rol activo de los emprendedores como agentes de cambio ha sido fundamental en la consolidación del ecosistema. Vale la pena destacar el papel fundamental de la política pública que no solo dio origen al Cluster mediante la creación de una Corporación sin ánimo de lucro que orienta las acciones de las empresas, sino que también disminuyó posteriormente la inyección de recursos ocasionando fuertes lesiones financieras y organizacionales que llevaron al cluster a una etapa de crisis. Posteriormente, los cambios de Gobierno y la adopción de políticas afines al desarrollo de la $\mathrm{CTel}$ en las regiones permiten que este tipo de iniciativas renazca con fuerza. Es la política pública un catalizador de todo el proceso, en donde, el rol de las organizaciones cobra importancia en la medida en que capitalicen los recursos de la coyuntura en la creación de una institucionalidad y tejido empresarial lo suficientemente fuertes para no depender de la intermitencia del recurso financiero estatal.

\section{Referencias bibliográficas}

Adner, R. (2006). Match your innovation strategy to your innovation ecosystem. Harvard Business Review, 84(4), 98-107.

Albach, H. (1967). Un modelo de comportamiento del crecimiento de la empresa. Boletín de Estudios Económicos. España.

Alvarado Muñoz, O., \& Rivera Martínez, W. (2011). Universidad y emprendimiento, aportes para la formación de profesionales emprendedores. Cuadernos de Administración (Universidad Del Valle), 27(45), 6174.

Blázquez, F., Dorta, J., \& Verona, M. (2006). Factores del crecimiento empresarial. Especial referencia a las empress pequeñas y medianas. Innovar, 16(28), 43-56.

Brüderl, J., \& Preisendörfer, P. (1998). Network support and the success of newly founded business. Small Business Economics, 10(3), 213225.

Bucheli, J. (2005). Aproximación al entorno empresarial payanes. Porik An, 23(12).

Gibrat, R. (1931). es inégalités économiques: applications, aux inégalitês des richesses, a la concentration des entreprises, aux populations des villes, aux statistiques des familles, etc.: d'une loi nouvelle la loi de l'effet proportionnel. Paris: Librarie du recueil sirey.

Hernández-Sampieri, R., Fernández, C., \& Baptista, M. (2014). Metodología de la investigación. México D.F: McGraw-Hill Education.

lansiti, M., \& Levien, R. (2004). The keystone advantage: what the new dynamics of business ecosystems 
mean for strategy, innovation, and sustainability. Harvard Business Press, 18(2), 15-75.

Lechner, C., \& Dowling, M. (2003). Firm networks: external relationships as sources for the groth and competitiveness of entrepreneurial firms. Entrepreneurship \& Regional Development, 15(1), 1-26.

Moore, I., \& Garnsey, E. (1993). Funding for innovation in small firms: The role of government. Research Policy, 22(5), 507-519.

OECD. (2014). Entrepreneurial ecosystems and growth oriented entrepreneurship. Netherlands.

Ostgaard, T., \& Birley, S. (1994). Personal networks and firm competitive strategy: a strategic or coincidential match? Journal of Business Venturing, 9(4), 281-305.

Ries, E. (2011). The Lean StartUp (3rd ed.). New York: Crown.
Sveiby, K. (1997). Capital intelectual: la nueva riqueza de las empresas. Cómo medir y gestionar los activos intangibles para crear valor. Gestión 2000.

Universidad Externado de Colombia. (2019). Tecnologías de la información en Bogotá. Bogotá D.C.

\section{Entrevistas}

Zambrano, Ricardo. Director Ejecutivo Cluster CreaTIC (2002-2006); Popayán 10 de enero de 2018

Santacruz, Guillermo. Director Ejecutivo Cluster CreaTIC (2007-2010); Popayán 15 de enero de 2018

Erazo, Jairo. Director Ejecutivo Cluster CreaTIC (2011-2014); Popayán 25 de enero de 2018

Samboní, Cesar. Director Ejecutivo Cluster CreaTIC (2014-2019); Popayán 10 de febrero de 2018

- $\quad$ Esta obra está bajo una licencia de Creative Commons Reconocimiento-NoComercial- Compartirlgual 3.0 Unported. http://creativecommons.org/licenses/by-nc-sa/3.0/deed.es_ES 